\title{
Review Article \\ Noninvasive Positive Pressure Ventilation in Chronic Heart Failure
}

\author{
Hao Jiang, ${ }^{1}$ Yi Han, ${ }^{2}$ Chenqi $\mathrm{Xu},{ }^{1,3}$ Jun $\mathrm{Pu}^{1}$ and Ben $\mathrm{He}^{1}$ \\ ${ }^{1}$ Department of Cardiovascular Diseases, Renji Hospital, Shanghai Jiao Tong University School of Medicine, Shanghai, China \\ ${ }^{2}$ Department of Geriatrics, First Affiliated Hospital of Nanjing Medical University, Nanjing 210029, China \\ ${ }^{3}$ Renal Division and Molecular Cell Laboratory for Kidney Disease, Renji Hospital, Shanghai Jiao Tong University School of Medicine, \\ Shanghai, China
}

Correspondence should be addressed to Jun Pu; pujun310@hotmail.com and Ben He; heben1025@hotmail.com

Received 3 June 2016; Revised 22 August 2016; Accepted 4 October 2016

Academic Editor: Zhongheng Zhang

Copyright (C) 2016 Hao Jiang et al. This is an open access article distributed under the Creative Commons Attribution License, which permits unrestricted use, distribution, and reproduction in any medium, provided the original work is properly cited.

\begin{abstract}
Instruction and Objectives. Noninvasive positive pressure ventilation (NPPV) alleviates sleep-disordered breathing (SDB) and it may improve cardiac function in SDB patients. Because large randomized controlled trials directly evaluating the impact of NPPV on cardiac function are lacking, we conducted a meta-analysis of published data on effectiveness of NPPV in improving cardiac function in patients with chronic heart failure regardless of SDB presence. Methods. Controlled trials were identified in PubMed, OVID, and EMBASE databases. Both fixed and randomized models were used in meta-analysis with primary outcomes of left ventricular ejection fraction (LVEF). Results. Nineteen studies were included with a total of 843 patients. Compared to standard medical treatment (SMT) plus sham-NPPV or SMT only, NPPV plus SMT was associated with improvement in LVEF (weighted mean difference $5.34,95 \%$ CI, $[3.85,6.82] ; P<0.00001$ ) and plasma brain natriuretic peptide (BNP) level (weighted mean difference -117.37, 95\% CI, [-227.22, -7.52]; $P=0.04$ ) and no influence on overall mortality (RR 1.00, 95\% CI, [0.96, 1.04]; $P=0.95$ ). Conclusions. In the present meta-analysis, use of NPPV plus SMT improved LVEF and reduced plasma BNP level but did not improve overall mortality in patients with chronic heart failure.
\end{abstract}

\section{Introduction}

According to an estimate by the American Heart Association (AHA), 5.1 million American adults suffered from heart failure (HF) in 2014 [1]. Although survival has improved over time, 5-year mortality of HF patients remains high at about $50 \%$ [2-4]. HF also poses a large financial burden on the healthcare system amounting in 2012 to approximately 20.9 million dollars in direct medical costs in the United States [5].

Forty percent to half of patients with chronic heart failure (CHF) and impaired left ventricular function go on to develop sleep-disordered breathing (SDB), either obstructive or central sleep apnea (OSA or CSA) [6-9], both of which disrupt the normal relaxing effects of sleep on the cardiovascular system. Accumulated evidence suggested that SDB accelerates the progression of CHF. SDB induces hypoxia and hypercapnia, promotes autonomic imbalance with sympathetic activation and parasympathetic inhibition, and increases the blood pressure and left ventricular afterload, all of which are stimuli to myocardial ischemia [1012], adverse cardiac remodeling [13-15], and left ventricular dysfunction $[16,17]$. Among the modalities of noninvasive positive pressure ventilation (NPPV) used to treat SDB in CHF patients, continuous positive airway pressure (cPAP) attenuates central sleep apnea, improves nocturnal oxygenation and left ventricular systolic function, and reduces excitability of the overactivated sympathetic nervous system [18-21]. The more recent adaptive servoventilation (ASV) also alleviates SDB and it may improve cardiac function in CHF patients [22-27].

Perhaps because of limitations in sample size, incomplete data reporting, and population differences, not all studies on NPPV have yielded positive results in terms of cardiac function improvement. For instance, Pepperell et al. found no difference in change in left ventricular ejection fraction (LVEF) between ASV treated patients and controls [25]; Egea 
et al. found no significant improvement in 6 min walking test between the CPAP and control groups [28]; and both Ferrier et al. and Hastings et al. found that neither cPAP nor ASV significantly decreased plasma BNP concentration $[29,30]$.

We therefore sought to explore in a meta-analysis if adult patients with CHF would benefit from NPPV in improving cardiac function, in the form of cPAP or ASV, as compared to standard medical treatment (SMT).

\section{Methods}

2.1. Search Strategy and Literature Screening. A systematic literature review was undertaken on January 26th, 2015, using PubMed, OVID, and EMBASE databases. To retrieve the largest number of potentially related studies, the following terms were used individually: "noninvasive positive pressure ventilation," "continuous positive airway pressure," "bilevel positive airway pressure," "adaptive servo-ventilation," and "heart failure." Articles were first screened by title and abstract, and reviews, meta-analyses, guidelines, letters, case reports, clinical trials in children, newborns, or postsurgical patients, and animal experiments were excluded. Three studies with full texts not written in English also were excluded.

The following criteria then were used to identify potentially suitable studies in a second screen: the trials were (a) well-designed randomized controlled trials (RCTs), quasirandomized controlled trials (qRCTs), and nonrandomized controlled trials; (b) enrolled subjects were adults older than 18 years and diagnosed with chronic heart failure, with or without sleep-disordered breathing (SDB); and (c) the intervention was noninvasive positive pressure ventilation (NPPV) in the form of continuous positive airway pressure (cPAP), adaptive servoventilation (ASV), or bilevel positive airway pressure (BiPAP), plus standard medical treatment (SMT), while the control treatment was SMT plus shamNPPV or SMT only; and (d) left ventricular ejection fraction (LVEF) must be included in the study outcomes.

After the above-mentioned screening, the authors obtained the full text articles and read them carefully and independently. Articles meeting the following criteria were excluded: (a) follow-up period was less than 4 weeks; (b) number of study participants was less than 10; (c) crossoverdesign was excluded if data before washout were not reported or unavailable; (d) outcome LVEF was only reported by a descriptive conclusion (original data or processed data were not reported or available); and (e) subjects from subgroup analysis of the other clinical trials were repeatedly counted. In addition, we excluded articles including BiPAP from the final analysis because BiPAP worsens, rather than improves, central apneas [31].

2.2. Data Extraction and Processing. Two authors extracted data independently. Descriptive data include first author, publication year, study design, duration of study arms, duration of washout (if applicable), type of control used, HF inclusion criteria, SDB inclusion criteria, proportion of male patients, mean age, and mean BMI (if available). For outcome data, the mean together with standard deviation
(SD) at baseline and end-trial time point was extracted for the NPPV and control arms. Standard error of the mean (SEM) was converted into SD. The change in mean was calculated as end-trial value minus baseline value. Variables reported in interquartile range were converted into mean using the method provided in the Cochrane Handbook for Systematic Reviews of Interventions (version 5.0.1) [32]. The change in SD was calculated using the formula also provided by the Cochrane Handbook for Systematic Reviews of Interventions (version 5.0.1) [32]. For the present meta-analysis, BNP was expressed in $\mathrm{pg} / \mathrm{mL}$ using the conversion factor provided by Weber and Hamm [33] when different units were used. Events were defined as refractory heart failure and worsening heart failure if (1) events reported as refractory heart failure, or (2) events reported as heart failure worsening after NPPV application, or (3) events reported as unstable need emergency transplantation and readmission due to heart failure worsening. In particular, for outcome in crossover studies, the mean and variability in the NPPV and control arms before washout were extracted (if available) in this article.

2.3. Data Analysis. Two authors (Chenqi Xu and Hao Jiang) conducted the analyses using Review Manager version 5.2 (Nordic Cochrane Center). The pooled estimate of mean weight difference (MWD) or risk ratio (RR) with their $95 \%$ $\mathrm{CI}$ was calculated using random effect model or fixed effect model according to heterogeneity among studies. $Q$ value and $I^{2}$ statistics, calculated when the number of analyzed studies exceeded three, were used as heterogeneity measures. A fixed effect model was used if there was no significant heterogeneity $\left(I^{2}<50 \%\right)$; otherwise, the random effects model would be applied for meta-analysis. A forest plot was constructed based on the results of pooled analysis of the NPPV and control arms. The primary outcome of this metaanalysis was LVEF. Subgroup analysis based on the degree of LVEF and reported geographical location was performed. Moreover, sensitivity analyses were performed to identify the effect of a single trial by sequential elimination of each trial from the pool and then to assess the overall outcomes. Statistical significance was set at $P$ value $<0.05$. Risk of bias was evaluated carefully and tabulated with brief details by Jun $\mathrm{Pu}$, M.D.

\section{Results}

3.1. Literature Search. In a search of the PubMed database, 1478 potential articles were identified. After applying the prespecified exclusion and inclusion criteria, the full texts of 75 articles were read, yielding 23 eligible studies. During data extraction and analysis, 4 additional studies were excluded for different reasons: Smith et al. (2007) [34] did not report the mean and variability before washout; Zhang et al. (2006) [35] conducted a trial over a period of 1 week, which was too short to meet the inclusion criteria; Gilman et al. (2008) [36] entailed a subgroup analysis of the CANPAP trial, that is, a redundant population; and Campbell et al. (2011)'s study included a population that was not large enough. Finally, 19 studies were included in the pooled analysis. The study 


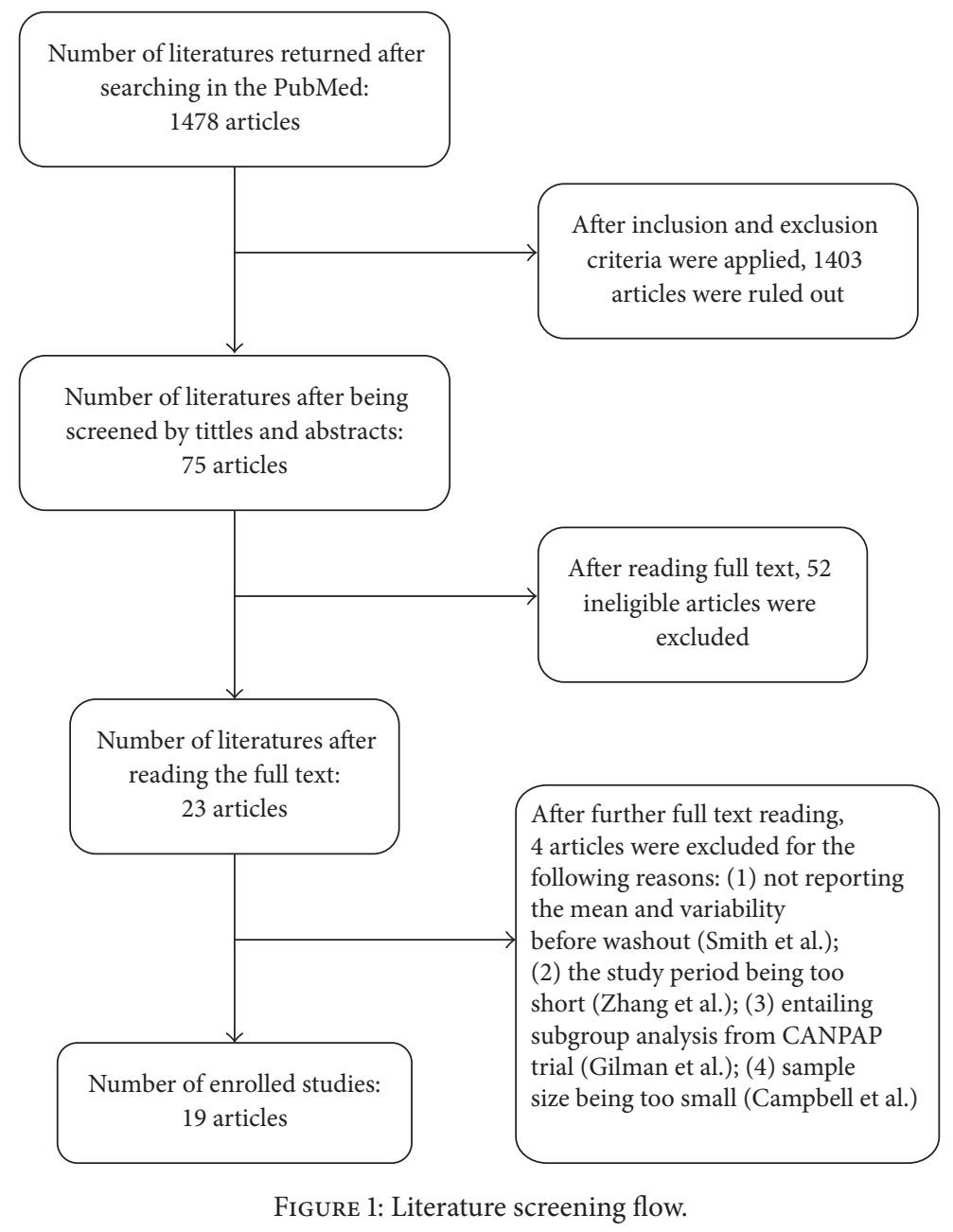

selection process is outlined in Figure 1. Characteristics of the included 19 studies are presented in Table 1. The risk of bias is presented in Figure 2. The included studies showed relatively high quality with an acceptable risk of bias overall (Figure 2(a)). However, there was high performance risk and detection bias in most of the studies (Figure 2(b)).

\subsection{Meta-Analysis}

3.2.1. Left Ventricular Ejection Fraction (LVEF). The weighted mean difference of the total is 5.34 favoring NPPV (95\% CI, [3.85, 6.82]; $P<0.00001)$. Heterogeneity between the studies was significant $(Q=41.0, P=0.002)$, and $I^{2}$ was $56 \%$. When the study by Bradley et al. (2005) [37] was removed from the pooled analysis, $I^{2}$ changed from $56 \%$ to $33 \%$.

The weighted mean difference of the cPAP subgroup was 3.85 favoring cPAP $(95 \% \mathrm{CI},[2.28,5.42] ; P<0.00001)$. Heterogeneity among studies in the cPAP subgroup was not significant $(Q=14.59, P=0.15)$, with $I^{2}$ of $31 \%$.

The weighted mean difference of the ASV subgroup was 6.83 favoring ASV (95\% CI, [4.46,9.19]; $P<0.00001)$. Heterogeneity among studies in the ASV subgroup was significant $\left(Q=15.11, P=0.03\right.$ ), with $I^{2}$ of $54 \%$ (see Figure 3).

The weighted mean difference of LVEF $<30 \%$ was 4.94 favoring NPPV (95\% CI, [2.78,7.10]; $P<0.00001)$. Heterogeneity among studies in the LVEF $<30 \%$ subgroup was significant $(Q=19.95, P=0.006)$, with $I^{2}$ of $65 \%$.

The weighted mean difference of LVEF $>30 \%$ was 5.73 favoring NPPV (95\% CI, [4.03, 7.44]; $P<0.00001)$. Heterogeneity among studies in the LVEF $>30 \%$ subgroup was not significant $(Q=12.39, P=0.26)$, with $I^{2}$ of $19 \%$.

The weighted mean difference of the European subgroup was 5.05 favoring ASV (95\% CI, [0.07, 10.03]; $P=0.05)$. Heterogeneity among studies in the ASV subgroup was significant $(Q=4.96, P=0.08)$, with $I^{2}$ of $60 \%$.

The weighted mean difference of the Asian subgroup was 7.92 favoring ASV (95\% CI, [5.58, 9.96]; $P=0.05)$. Heterogeneity among studies in the ASV subgroup was not significant $(Q=2.43, P<0.00001)$, with $I^{2}$ of $8.7 \%$.

3.2.2. Left Ventricular End-Diastolic Dimension (LVEDD). Five studies reported data on change in LVEDD between before and after intervention. The weighted mean difference 


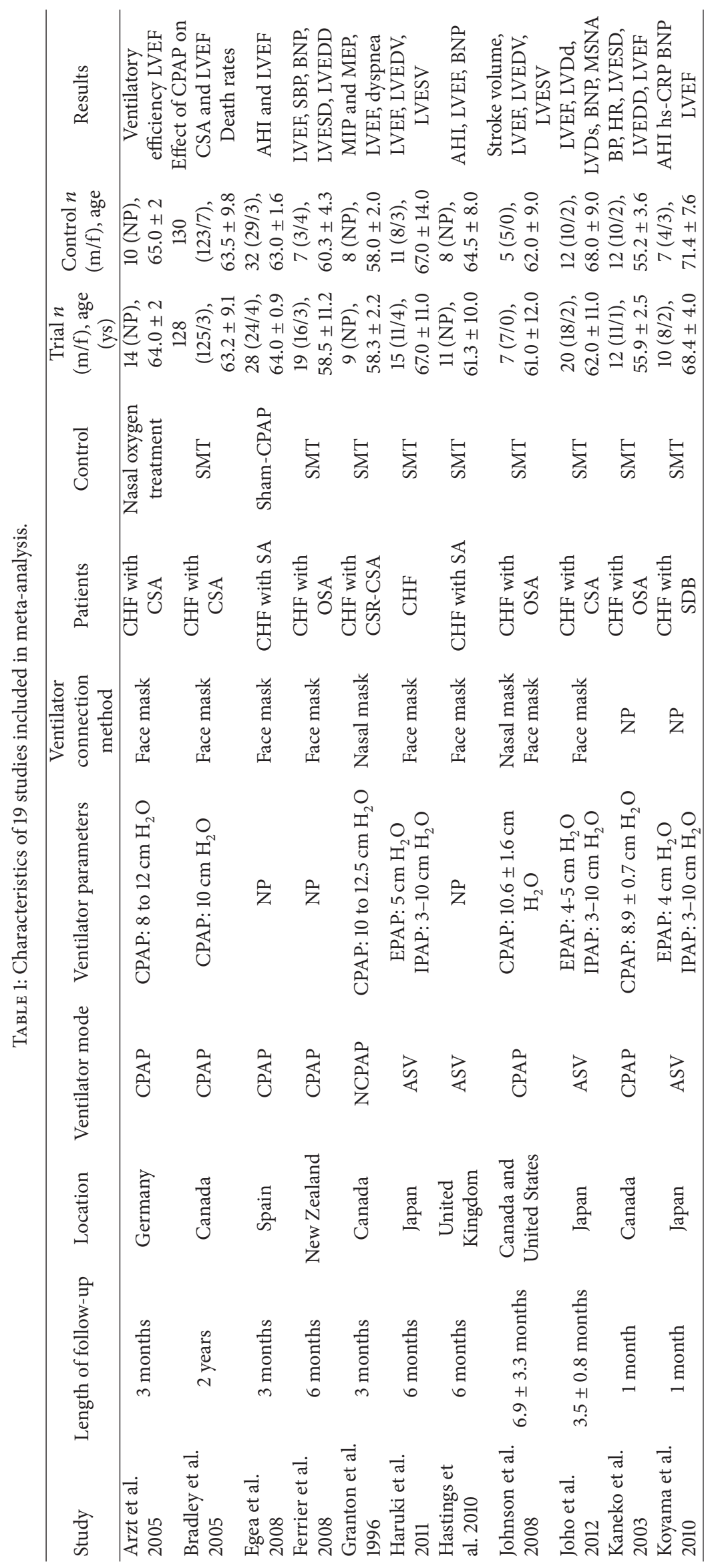




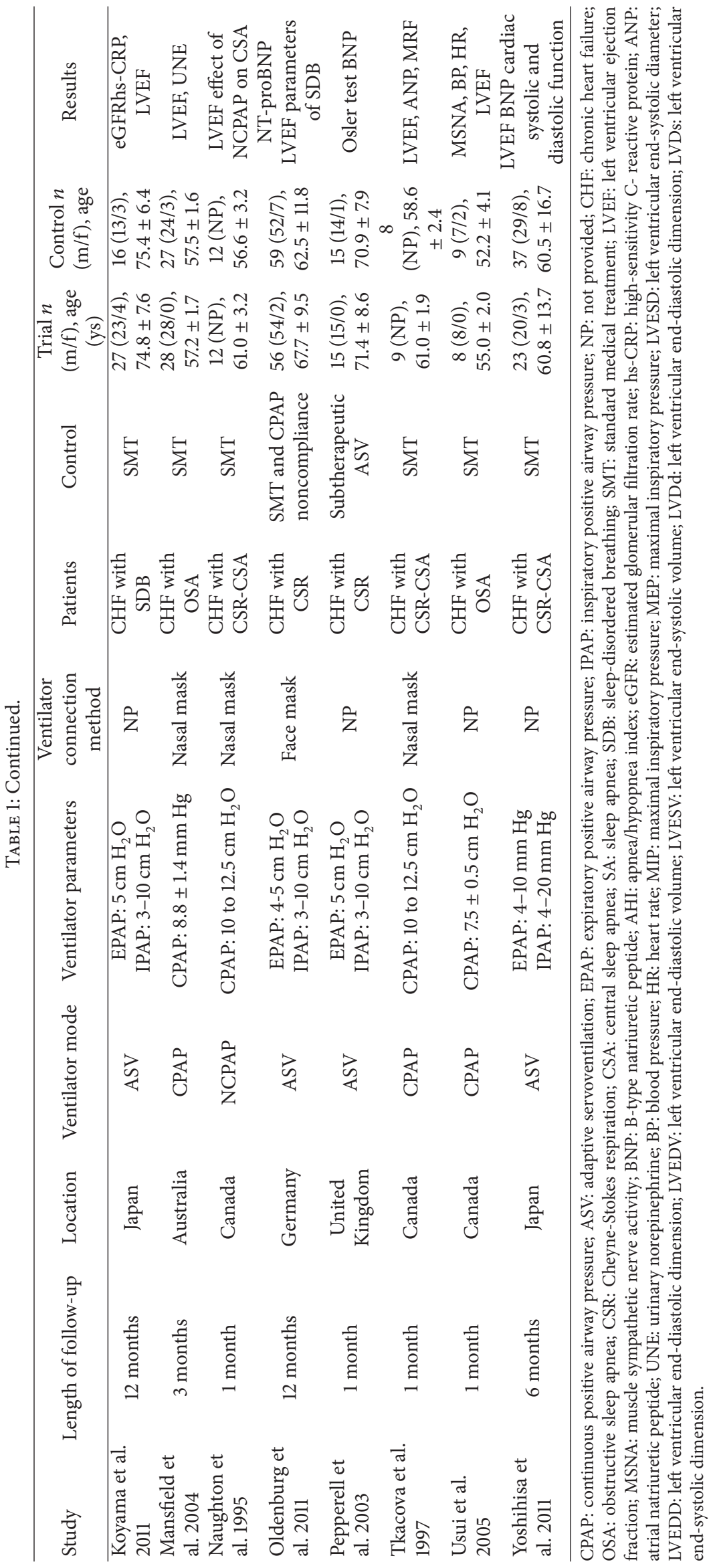




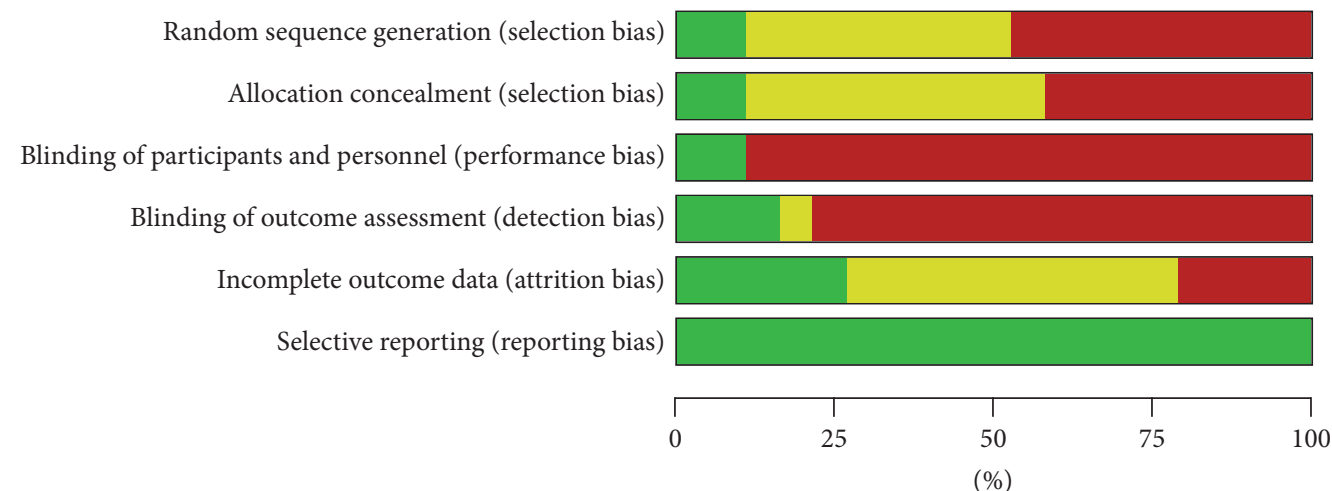

Low risk of bias $\square$ High risk of bias

Unclear risk of bias

(a)

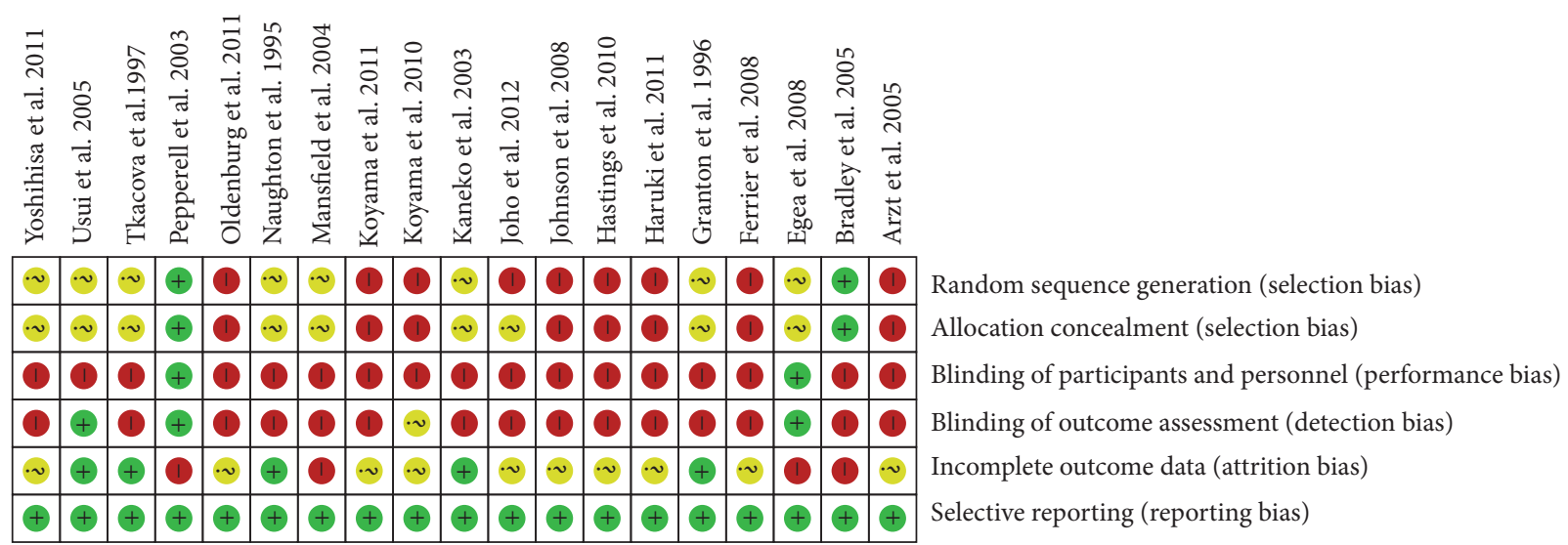

(b)

FIGURE 2: Risk of bias of the included studies. (a) Risk of bias graph; (b) risk of bias summary.

of the total was -1.91 favoring NPPV (95\% CI, [-4.60, 0.78]; $P=0.16)$. Heterogeneity among studies was not significant $(Q=8.13, P=0.09)$.

The weighted mean difference of the cPAP subgroup was 0.45 favoring control $(95 \% \mathrm{CI},[-6.0,6.89] ; P=0.89)$. Heterogeneity among studies in the cPAP subgroup was significant $(Q=3.79, P=0.05)$, with $I^{2}$ of $74 \%$.

The weighted mean difference of the ASV subgroup was -3.60 favoring ASV (95\% CI, $[-5.19,-1.50] ; P=0.0008)$. Heterogeneity among studies in the ASV subgroup was small $(Q=1.39, P=0.5)$, with $I^{2}$ of $0 \%$.

3.2.3. Plasma BNP Level. Six studies reported data on plasma BNP level before and after intervention; 5 from the ASV subgroup and one from the cPAP subgroup. The weighted mean difference of the total was -117.37 favoring NPPV $(95 \%$ CI, $[-227.22,-7.52] ; P=0.04)$. Heterogeneity among studies was significant $(Q=26.40, P<0.0001)$, with $I^{2}$ of $81 \%$.

The mean difference of the cPAP subgroup was 4.50, not significantly favoring the control (95\% CI, [-77.12, 86.12]; $P=0.91)$. And the weighted mean difference of the ASV subgroup was -152.58 favoring ASV (95\% CI, [-295.81, -9.35];
$P=0.04)$. Heterogeneity among studies in the ASV subgroup was significant $(Q=26.40, P=0.0001)$, with $I^{2}$ of $83 \%$.

3.2.4. Overall Mortality. In 19 trials involving 913 patients, we did not find difference in overall mortality between patients treated with NPPV plus standard medical treatment (SMT) and with SMT alone (RR 1.00, 95\% CI, [0.96, 1.04]; $P=0.95$ ) (Figure 4).

3.2.5. Adverse Events. Refractory heart failure and worsening heart failure: of the 19 studies included, 6 reported the events as defined. We found no difference in the incidence of refractory heart failure and worsening heart failure between patients treated with NPPV plus SMT and SMT alone (RR $1.07,95 \%$ CI, [0.95, 1.21]; $P=0.25$ ).

Cardiac arrest: 3 studies reported the incidence of cardiac arrest and we found no difference in the incidence of cardiac arrest between the two groups (RR 1.02, 95\% CI, [0.93, 1.12]; $P=0.63)$.

Angina and acute myocardial infarction (AMI): 3 studies reported the incidence of angina and AMI. We found no difference in the incidence of angina and AMI between the two groups (RR 1.01, 95\% CI, [0.95, 1.08]; $P=0.64$ ). 


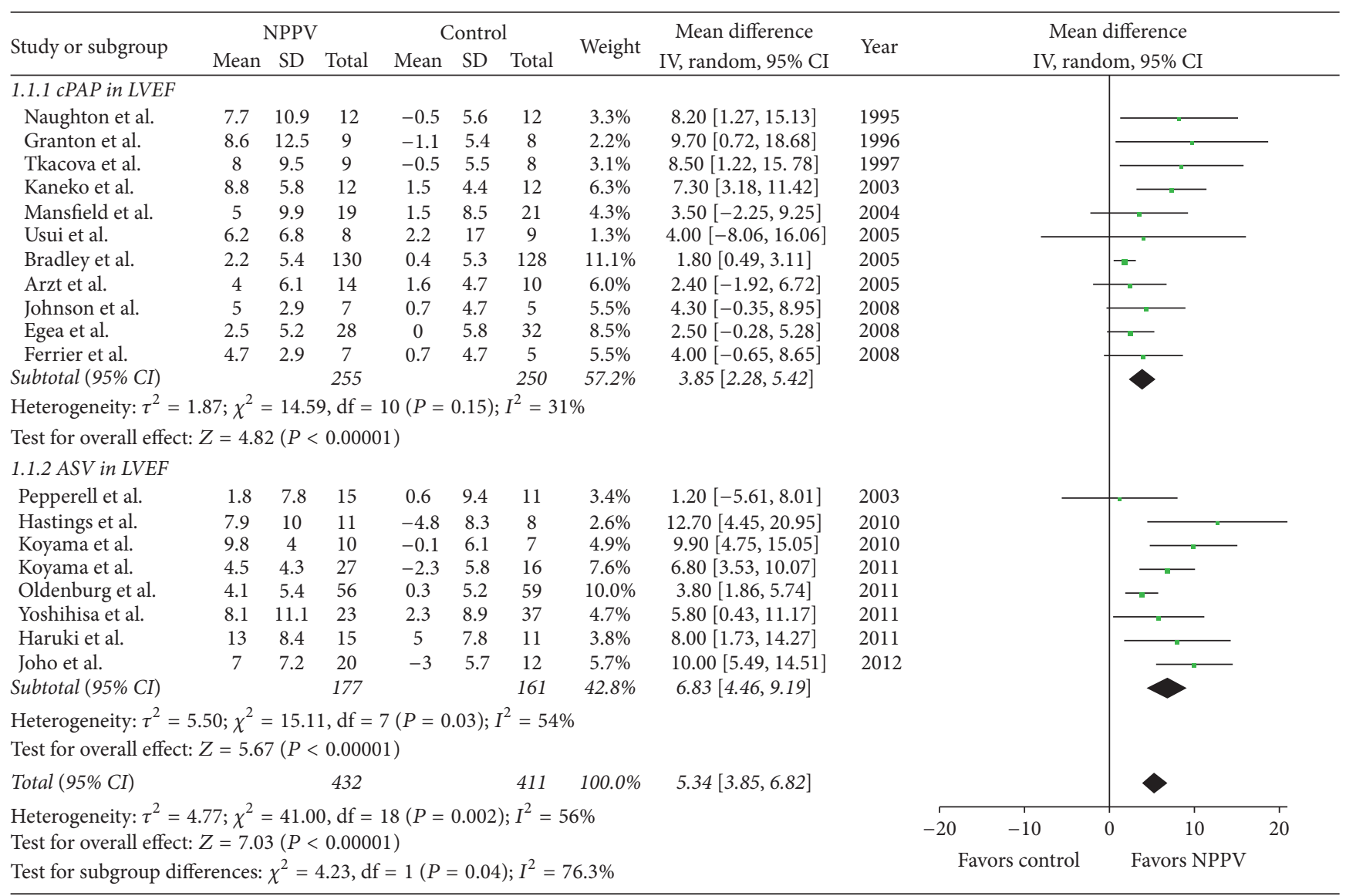

FIGURE 3: Forest plot of the effect of noninvasive positive airway pressure (cPAP and ASV) therapy for chronic heart failure on left ventricular ejection fraction (LVEF). CI: confidence interval; IV: inverse variance; SD: standard deviation; MD: mean difference.

3.2.6. Sensitivity Analysis. Sensitivity analyses by sequentially dropping individual trials and then evaluating the overall outcomes failed to identify any of the individual trials as having influenced the primary outcomes of the present metaanalysis to a significant extent (Table 2).

\section{Discussion}

Two main conclusions can be drawn for the present metaanalysis. Firstly, NPPV plus standard medical treatment (SMT) improved LVEF but did not improve overall mortality. Secondly, relative to SMT plus sham-NPPV/SMT alone, NPPV improved plasma BNP level but did not improve LVEDD and decrease threats of cardiac arrest events, angina, and AMI events.

\subsection{Primary Outcomes}

4.1.1. NPPV Improves LVEF in Chronic Heart Failure Patients. The present meta-analysis revealed that NPPV improves cardiac function by increasing LVEF. Among included studies, the majority of patients already had reduced LVEF or were in the course of developing heart failure with reduced LVEF and therefore were considered more likely to benefit from the use of NPPV. The results are consistent with those of many previous studies [19, 20, 30, 37-45]. However, several studies $[21,25,28,29,46-48]$, most nonrandomized and with small sample sizes, showed that NPPV had no significant effects on the improvement of the cardiac function.

In our analyses, the weighted mean difference of the cPAP subgroup was 3.85 favoring cPAP, while that of the ASV subgroup was 6.83 favoring ASV. This might indicate that ASV is better than CPAP in the improvement of LVEF. However, the conclusion did not come from the direct comparison of cPAP and ASV since none of the included studies presented such a direct comparison. Interestingly, two randomized controlled trials showed that $\mathrm{CHF}$ patients with SDB might gain greater benefit from treatment with ASV than with CPAP $[26,49]$, which was consistent with our results.

The total heterogeneity of the aforementioned part of the analysis is significant (Figure 5). In the cPAP subgroup, the subtotal heterogeneity is not significant $\left(I^{2}=31 \%\right)$, while that of ASV subgroup is significant $\left(I^{2}=54 \%\right)$. To gain further insight into the heterogeneity, we performed subgroup analyses. Firstly, we used LVEF $<30 \%$ and LVEF $>30 \%$ as grouping criteria. Then, we found that when the mean LVEF of the NPPV group before intervention exceeded $30 \%$, the weighted mean difference was favoring NPPV and the heterogeneity was small. However, when the mean LVEF of the NPPV group 


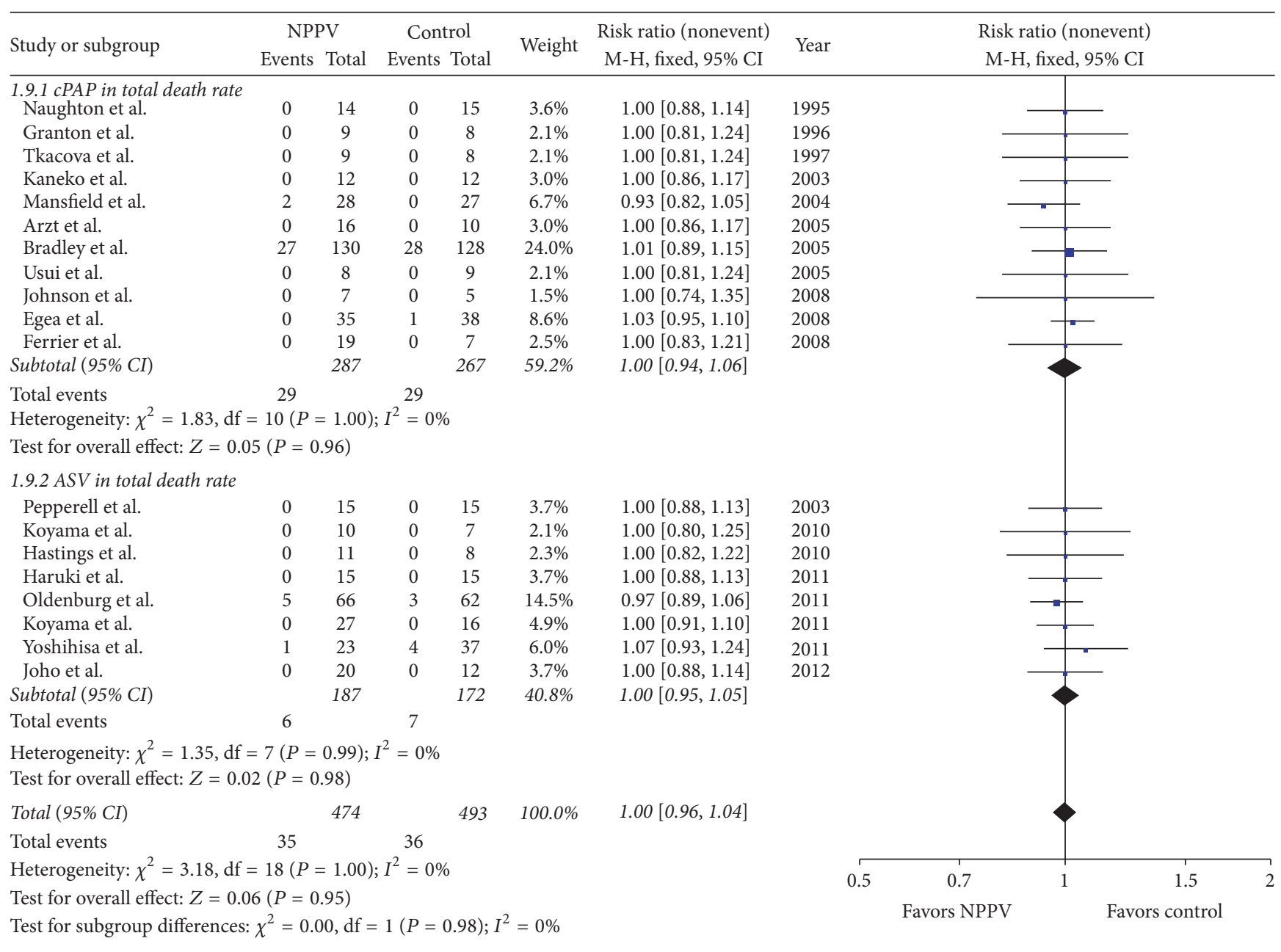

FIGURE 4: Forest plot of the effect of noninvasive positive airway pressure (cPAP and ASV) therapy for chronic heart failure on total mortality. $\mathrm{CI}$ : confidence interval; $\mathrm{M}-\mathrm{H}$ : inverse variance; RR: risk ratio.

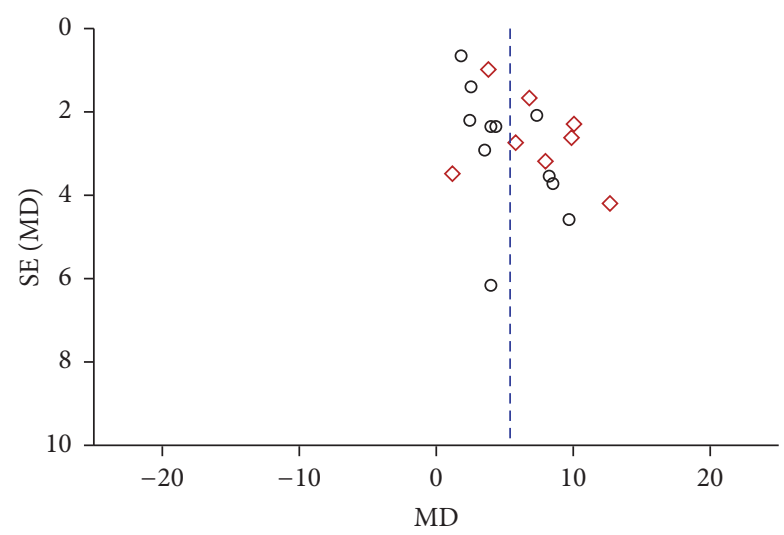

Subgroups

CPAP in LVEF

$\diamond \mathrm{ASV}$ in LVEF

FIGURE 5: Funnel plot of NPPV on LVEF.

before intervention was $30 \%$ or less, the heterogeneity was significant. The latter result probably indicated a worse status among enrolled subjects with an LVEF of 30\% or less, in turn leading to a worse prognosis and underlying a statistically significant heterogeneity.

Secondly, we analyzed the difference in LVEF-change among study regions for the ASV subgroup. According to the reported geographical location where the study was conducted, we divided the 8 studies into the European subgroup (3 studies, 2 in UK, and 1 in Spain) and Asian subgroup (5 studies, all in Japan). We found that the Asian subgroup's heterogeneity was small $\left(Q=2.43, I^{2}=0 \%\right)$, while the heterogeneity of the European subgroup was significant $\left(Q=4.96, I^{2}=60 \%\right)$. The regional disparity, the difference between medical care systems, and even the different races of patients may underlie the observations in the present study, which warrant further study.

4.1.2. NPPV Did Not Improve Mortality. According to the result of the analysis, the use of NPPV plus SMT did not improve overall mortality among patients with chronic heart failure. The analysis showed good homogeneity among all 19 studies enrolled $\left(Q=3.18, I^{2}=0 \%\right.$ ) (Figure 6). Moreover, NPPV did not decrease cardiac adverse events in patients 
TABLE 2: Sensitivity analysis showing the effect sizes for the primary outcomes after removing individual trials included in the meta-analysis.

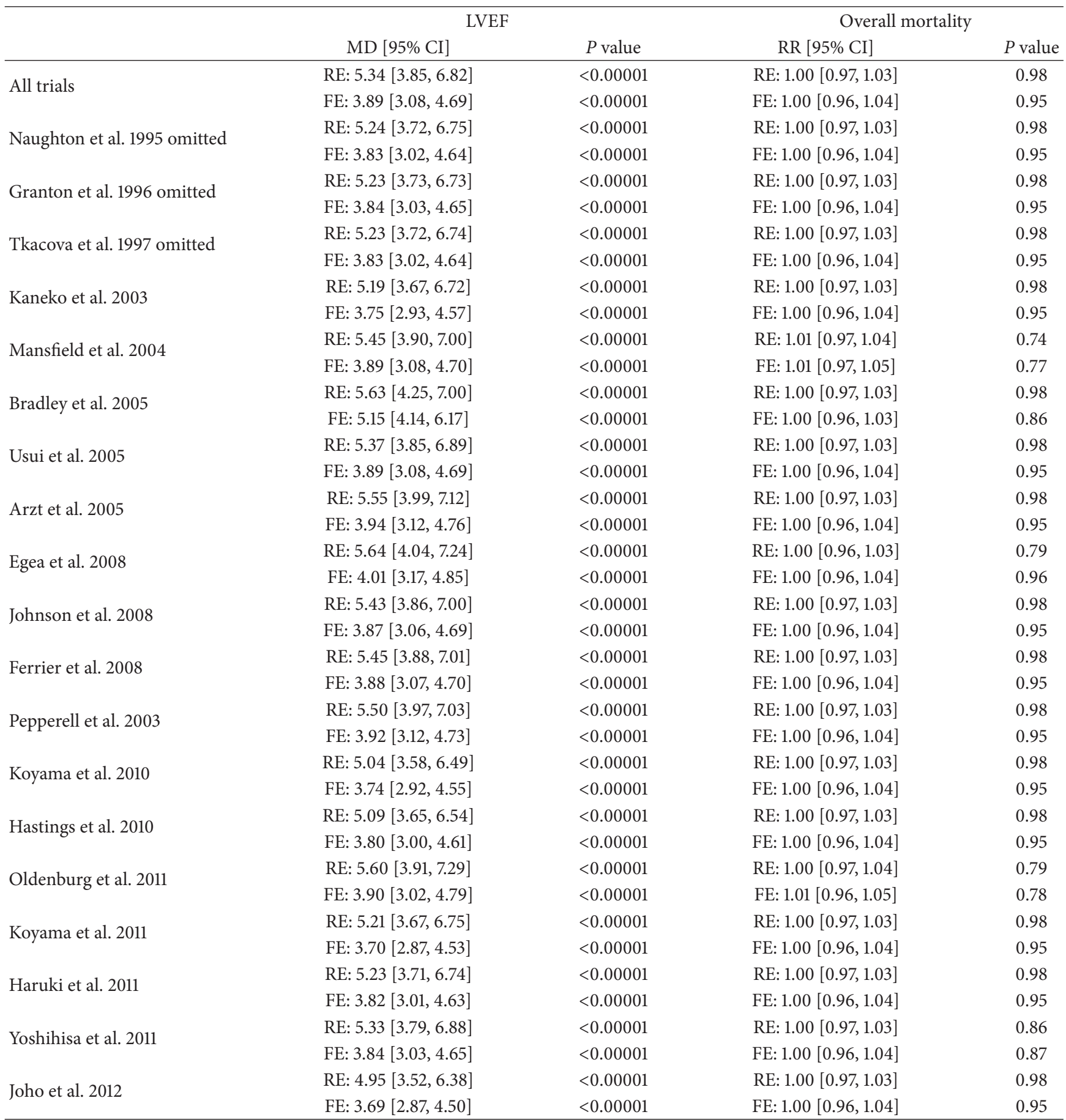

LVEF: left ventricular ejection fraction; MD: mean difference; RR: risk ratio; RE: random effect model; FE: fixed effect model.

with chronic heart failure according to analysis of adverse events (including refractory heart failure and worsening heart failure, cardiac arrest, and angina and acute myocardial infarction), with good homogeneity among all these analyses.

Despite the aforementioned results, the impact of NPPV on overall mortality and cardiac adverse events remains to be further investigated. The longest follow-up period among the 19 studies was only 12 months and the shortest was 4 weeks. In a single center cohort study in Canada, patients with OSA were followed up for a decade; however, the study unfortunately did not provide information on cPAP use [50]. In a recent study (which was not included in the present analysis because of the lack of LVEF data), ASV increased the overall and cardiovascular mortality in CHF patients with 


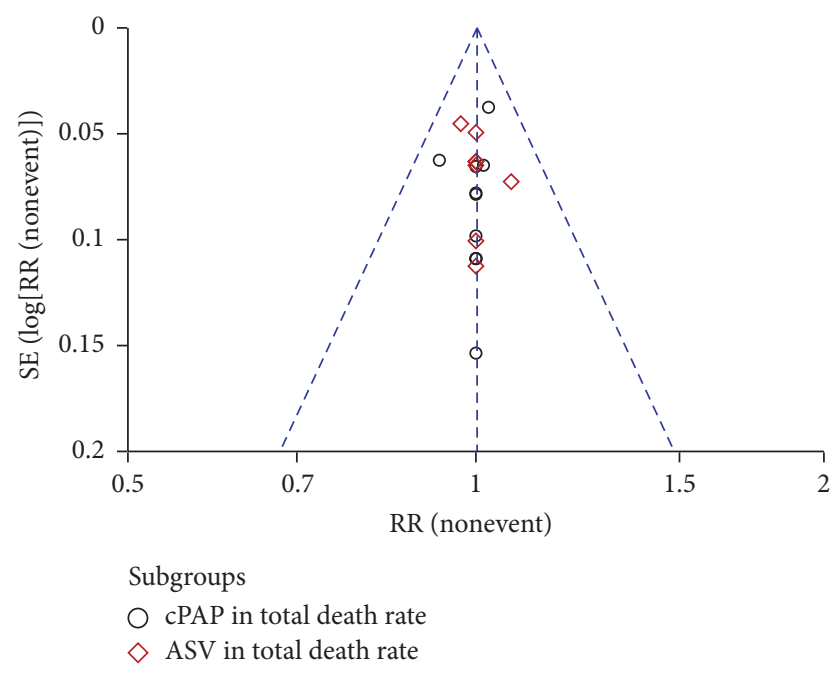

FIGURE 6: Funnel plot of NPPV on total mortality.

OSA [51]. Thus, more studies are warranted to evaluate the effect of NPPV on 3-year, 5-year, and even 10-year mortality rate.

\subsection{Other Secondary Outcomes}

4.2.1. NPPV Did Not Reduce LVEDD, but Results of Subgroup Analyses Differed. Five studies reported changes in LVEDD. The present analysis showed that NPPV did not reduce LVEDD; however, heterogeneity was significant. Subgroup analysis, however, yielded a different result. The weighted mean difference was 0.45 favoring (not significantly) control $(P=0.89)$ in the cPAP subgroup, while it was -3.60 favoring ASV $(P=0.0008)$ in the ASV subgroup, indicating that ASV might do better in reducing LVEDD than CPAP, although there was no direct comparison between CPAP and ASV. Further studies are warranted on LVEDD according to NPPV modality.

4.2.2. NPPV Reduces Plasma BNP Level in Patients with Chronic Heart Failure. Among included studies, 6 reported the plasma BNP level at baseline and after intervention. One study focuses on cPAP, while the other five studies focus on ASV. The present analysis showed that the use of NPPV reduced plasma BNP level in CHF patients. Because BNP level can be used to indicate prognosis and predict mortality and clinical outcome of patients with chronic heart failure $[33,52]$, the reduced BNP level may indicate a better prognosis. However, the subgroup analysis showed no significant difference between CPAP and SMT in influencing plasma BNP level. Since there is only one cPAP study involved, the conclusion may be not applicable to cPAP. Conversely, ASV showed effectiveness in reducing BNP level, possibly indicating that ASV might improve the clinical outcome of CHF patients and reduce mortality. However, the heterogeneity of the analysis was very significant, and further studies are therefore warranted.
ASV was designed to meet the patients' ventilation support by providing inspiratory positive airway pressure (IPAP) and adjust the rate of change of airflow through sensing the patient airflow. cPAP, however, provided a continuous pressure which could not be adjusted according to the patients breath [53]. Several studies showed ASV was associated with significantly better compliance when compared with cPAP [49]. Interestingly, ASV was found to increase 1-year survival rate and reduce cardiovascular events in $\mathrm{CHF}$ patient, while cPAP did not show survival benefit among patients with CSA [37].

4.3. Study Limitations. Our study has several potential limitations. First, the sample sizes of component trials included in our analysis are generally not large, which may bring "smallstudy effects." "Small-study effects" refer to the fact that trials with limited sample sizes are more likely to report larger beneficial effects than large trials $[54,55]$. Thus, we performed sensitivity analyses to test the impact of individual trials on the overall result of meta-analysis. Second, only two studies included in our meta-analysis presented the data on NPPV compliance. Ferrier et al. pointed out patients using CPAP $(>1 \mathrm{~h}$ per night CPAP) had the greatest increase in LVEF [29]; Joho et al. found that the change in average use of ASV correlated with changes in LVEF [41]. However, the definition of NPPV compliance in those reports was not consistent and the influence of compliance to treatment was not quantified. Thus, we did not report the influence of NPPV compliance on studied variables in the present study.

\section{Conclusions}

In the present meta-analysis, relative to SMT plus shamNPPV/SMT alone, NPPV plus SMT improved LVEF and reduced plasma BNP level but did not improve overall mortality and adverse event rates.

\section{Abbreviations \\ NPPV: Noninvasive positive pressure ventilation \\ cPAP: Continuous positive airway pressure \\ ASV: $\quad$ Adaptive servoventilation \\ BiPAP: Bilevel positive airway pressure \\ SDB: $\quad$ Sleep-disordered breathing \\ OSA: Obstructive sleep apnea \\ CSA: Central sleep apnea \\ CSR: Cheyne-Stokes respiration \\ LVEF: Left ventricular ejection fraction \\ LVEDD: Left ventricular end-diastolic dimension \\ LVESD: Left ventricular end-systolic dimension \\ BNP: $\quad$ Brain natriuretic peptide \\ Peak $\mathrm{VO}_{2}$ : Peak oxygen consumption.}

\section{Competing Interests}

The authors have no financial disclosures or competing interests to declare. 


\section{Authors' Contributions}

Hao Jiang and Yi Han contributed equally to this work.

\section{Acknowledgments}

This work was supported by the National Science Fund for Distinguished Young Scholars (81625002 to Jun Pu), Program for New Century Excellent Talents of Ministry of Education of China (NCET-12-0352), Shanghai Shuguang Program (12SG22), Program of Shanghai Committee of Science and Technology (15411963600 and 14JC1404500), Shanghai Municipal Education Commission Gaofeng Clinical Medicine Grant Support (20152209), Shanghai Jiaotong University (YG2013MS420), and Shanghai Jiao Tong University School of Medicine (15ZH1003 and 14XJ10019).

\section{References}

[1] A. S. Go, D. Mozaffarian, V. L. Roger et al., "Heart disease and stroke statistics-2014 update: a report from the American Heart Association," Circulation, vol. 129, no. 3, pp. e28-e292, 2014.

[2] V. L. Roger, S. A. Weston, M. M. Redfield et al., "Trends in heart failure incidence and survival in a community-based population," The Journal of the American Medical Association, vol. 292, no. 3, pp. 344-350, 2004.

[3] D. Levy, S. Kenchaiah, M. G. Larson et al., "Long-term trends in the incidence of and survival with heart failure," The New England Journal of Medicine, vol. 347, no. 18, pp. 1397-1402, 2002.

[4] F. Simko, K. R. Bednarova, K. Krajcirovicova et al., "Melatonin reduces cardiac remodeling and improves survival in rats with isoproterenol-induced heart failure," Journal of Pineal Research, vol. 57, no. 2, pp. 177-184, 2014.

[5] P. A. Heidenreich, N. M. Albert, L. A. Allen et al., "Forecasting the impact of heart failure in the United States: a policy statement from the American Heart Association," Circulation: Heart Failure, vol. 6, no. 3, pp. 606-619, 2013.

[6] S. Javaheri, T. J. Parker, L. Wexler et al., "Occult sleep-disordered breathing in stable congestive heart failure," Annals of Internal Medicine, vol. 122, no. 7, pp. 487-492, 1995.

[7] D. D. Sin, F. Fitzgerald, J. D. Parker, G. Newton, J. S. Floras, and T. D. Bradley, "Risk factors for central and obstructive sleep apnea in 450 men and women with congestive heart failure," American Journal of Respiratory and Critical Care Medicine, vol. 160, no. 4, pp. 1101-1106, 1999.

[8] M. Christ, Y. Sharkova, H. Fenske et al., "Brain natriuretic peptide for prediction of Cheyne-Stokes respiration in heart failure patients," International Journal of Cardiology, vol. 116, no. 1, pp. 62-69, 2007.

[9] H.-M. Yeung, M.-W. Hung, C.-F. Lau, and M.-L. Fung, "Cardioprotective effects of melatonin against myocardial injuries induced by chronic intermittent hypoxia in rats," Journal of Pineal Research, vol. 58, no. 1, pp. 12-25, 2015.

[10] Y. Yang, Y. Sun, W. Yi et al., "A review of melatonin as a suitable antioxidant against myocardial ischemia-reperfusion injury and clinical heart diseases," Journal of Pineal Research, vol. 57, no. 4, pp. 357-366, 2014.
[11] L. Yu, H. Liang, X. Dong et al., "Reduced silent information regulator 1 signaling exacerbates myocardial ischemiareperfusion injury in type 2 diabetic rats and the protective effect of melatonin," Journal of Pineal Research, vol. 59, no. 3, pp. 376-390, 2015.

[12] L. Yu, Y. Sun, L. Cheng et al., "Melatonin receptor-mediated protection against myocardial ischemia/reperfusion injury: role of SIRT1," Journal of Pineal Research, vol. 57, no. 2, pp. 228-238, 2014.

[13] X. Zhu, J. Fang, D.-S. Jiang et al., "Exacerbating pressure overload-induced cardiac hypertrophy: novel role of adaptor molecule src homology 2-B3," Hypertension, vol. 66, no. 3, pp. 571581, 2015.

[14] L. Gao, K. Huang, D.-S. Jiang et al., "Novel role for caspaseactivated DNase in the regulation of pathological cardiac hypertrophy," Hypertension, vol. 65, no. 4, pp. 871-881, 2015.

[15] R. Gogiraju, X. Xu, M. L. Bochenek et al., "Endothelial p53 deletion improves angiogenesis and prevents cardiac fibrosis and heart failure induced by pressure overload in mice," Journal of the American Heart Association, vol. 4, no. 2, Article ID e001770, 2015.

[16] T. D. Bradley and J. S. Floras, "Sleep apnea and heart failure: part I: obstructive sleep apnea," Circulation, vol. 107, no. 12, pp. 1671-1678, 2003.

[17] T. D. Bradley and J. S. Floras, "Sleep apnea and heart failure: part II: central sleep apnea," Circulation, vol. 107, no. 13, pp. 18221826, 2003.

[18] M. T. Naughton, D. C. Benard, P. P. Liu, R. Rutherford, F. Rankin, and T. D. Bradley, "Effects of nasal CPAP on sympathetic activity in patients with heart failure and central sleep apnea," American Journal of Respiratory and Critical Care Medicine, vol. 152, no. 2, pp. 473-479, 1995.

[19] M. T. Naughton, P. P. Liu, D. C. Benard, R. S. Goldstein, and T. D. Bradley, "Treatment of congestive heart failure and CheyneStokes respiration during sleep by continuous positive airway pressure," American Journal of Respiratory and Critical Care Medicine, vol. 151, no. 1, pp. 92-97, 1995.

[20] Y. Kaneko, J. S. Floras, K. Usui et al., "Cardiovascular effects of continuous positive airway pressure in patients with heart failure and obstructive sleep apnea," The New England Journal of Medicine, vol. 348, no. 13, pp. 1233-1241, 2003.

[21] K. Usui, T. D. Bradley, J. Spaak et al., "Inhibition of awake sympathetic nerve activity of heart failure patients with obstructive sleep apnea by nocturnal continuous positive airway pressure," Journal of the American College of Cardiology, vol. 45, no. 12, pp. 2008-2011, 2005.

[22] T. Kasai, K. Narui, T. Dohi et al., "First experience of using new adaptive servo-ventilation device for Cheyne-Stokes respiration with central sleep apnea among Japanese patients with congestive heart failure-report of 4 clinical cases," Circulation Journal, vol. 70, no. 9, pp. 1148-1154, 2006.

[23] M. Arzt, R. Wensel, S. Montalvan et al., "Effects of dynamic bilevel positive airway pressure support on central sleep apnea in men with heart failure," Chest, vol. 134, no. 1, pp. 61-66, 2008.

[24] W. J. Randerath, W. Galetke, S. Stieglitz, C. Laumanns, and T. Schäfer, "Adaptive servo-ventilation in patients with coexisting obstructive sleep apnoea/hypopnoea and Cheyne-Stokes respiration," Sleep Medicine, vol. 9, no. 8, pp. 823-830, 2008. 
[25] J. C. T. Pepperell, N. A. Maskell, D. R. Jones et al., "A randomized controlled trial of adaptive ventilation for cheyne-stokes breathing in heart failure," American Journal of Respiratory and Critical Care Medicine, vol. 168, no. 9, pp. 1109-1114, 2003.

[26] C. Philippe, M. Stoïca-Herman, X. Drouot et al., "Compliance with and effectiveness of adaptive servoventilation versus continuous positive airway pressure in the treatment of CheyneStokes respiration in heart failure over a six month period," Heart, vol. 92, no. 3, pp. 337-342, 2006.

[27] O. Oldenburg, A. Schmidt, B. Lamp et al., "Adaptive servoventilation improves cardiac function in patients with chronic heart failure and Cheyne-Stokes respiration," European Journal of Heart Failure, vol. 10, no. 6, pp. 581-586, 2008.

[28] C. J. Egea, F. Aizpuru, J. A. Pinto et al., "Cardiac function after CPAP therapy in patients with chronic heart failure and sleep apnea: a multicenter study," Sleep Medicine, vol. 9, no. 6, pp. 660-666, 2008.

[29] K. A. Ferrier, A. M. Neill, T. O’Meeghan, M. Richards, and A. J. Campbell, "Continuous positive airway pressure in heart failure patients with obstructive sleep apnoea," Internal Medicine Journal, vol. 38, no. 11, pp. 829-836, 2008.

[30] P. C. Hastings, A. Vazir, G. E. Meadows et al., "Adaptive servoventilation in heart failure patients with sleep apnea: a real world study," International Journal of Cardiology, vol. 139, no. 1, pp. 17-24, 2010.

[31] K. G. Johnson and D. C. Johnson, "Bilevel positive airway pressure worsens central apneas during sleep," Chest, vol. 128, no. 4, pp. 2141-2150, 2005.

[32] J. P. T. Higgins and S. Green, Cochrane Handbook for Systematic Reviews of Interventions, John Wiley \& Sons, 2008.

[33] M. Weber and C. Hamm, "Role of B-type natriuretic peptide (BNP) and NT-PROBNP in clinical routine," Heart, vol. 92, no. 6, pp. 843-849, 2006.

[34] L. A. Smith, M. Vennelle, R. S. Gardner et al., "Auto-titrating continuous positive airway pressure therapy in patients with chronic heart failure and obstructive sleep apnoea: a randomized placebo-controlled trial," European Heart Journal, vol. 28, no. 10, pp. 1221-1227, 2007.

[35] X.-L. Zhang, K.-S. Yin, X.-L. Li, E.-Z. Jia, and M. Su, "Efficacy of adaptive servoventilation in patients with congestive heart failure and Cheyne-Stokes respiration," Chinese Medical Journal, vol. 119, no. 8, pp. 622-627, 2006.

[36] M. P. Gilman, J. S. Floras, K. Usui, Y. Kaneko, R. S. T. Leung, and T. D. Bradley, "Continuous positive airway pressure increases heart rate variability in heart failure patients with obstructive sleep apnoea," Clinical Science, vol. 114, no. 3, pp. 243-249, 2008.

[37] T. D. Bradley, A. G. Logan, R. J. Kimoff et al., "Continuous positive airway pressure for central sleep apnea and heart failure," The New England Journal of Medicine, vol. 353, no. 19, pp. 2025-2033, 2005.

[38] T. Koyama, H. Watanabe, S. Terada et al., "Adaptive servo-ventilation improves renal function in patients with heart failure," Respiratory Medicine, vol. 105, no. 12, pp. 1946-1953, 2011.

[39] J. T. Granton, M. T. Naughton, D. C. Benard, P. P. Liu, R. S. Goldstein, and T. D. Bradley, "CPAP improves inspiratory muscle strength in patients with heart failure and central sleep apnea," American Journal of Respiratory and Critical Care Medicine, vol. 153, no. 1, pp. 277-282, 1996.

[40] N. Haruki, M. Takeuchi, K. Kaku et al., "Comparison of acute and chronic impact of adaptive servo-ventilation on left chamber geometry and function in patients with chronic heart failure," European Journal of Heart Failure, vol. 13, no. 10, pp. 1140-1146, 2011.

[41] S. Joho, Y. Oda, R. Ushijima, T. Hirai, and H. Inoue, "Effect of adaptive servoventilation on muscle sympathetic nerve activity in patients with chronic heart failure and central sleep apnea," Journal of Cardiac Failure, vol. 18, no. 10, pp. 769-775, 2012.

[42] T. Koyama, H. Watanabe, Y. Kobukai et al., "Beneficial effects of adaptive servo ventilation in patients with chronic heart failure," Circulation Journal, vol. 74, no. 10, pp. 2118-2124, 2010.

[43] O. Oldenburg, T. Bitter, R. Lehmann et al., "Adaptive servoventilation improves cardiac function and respiratory stability," Clinical Research in Cardiology, vol. 100, no. 2, pp. 107-115, 2011.

[44] R. Tkacova, P. P. Liu, M. T. Naughton, and T. D. Bradley, "Effect of continuous positive airway pressure on mitral regurgitant fraction and atrial natriuretic peptide in patients with heart failure," Journal of the American College of Cardiology, vol. 30, no. 3, pp. 739-745, 1997.

[45] A. Yoshihisa, T. Shimizu, T. Owada et al., "Adaptive servo ventilation improves cardiac dysfunction and prognosis in chronic heart failure patients with cheyne-stokes respiration," International Heart Journal, vol. 52, no. 4, pp. 218-223, 2011.

[46] M. Arzt, M. Schulz, R. Wensel et al., "Nocturnal continuous positive airway pressure improves ventilatory efficiency during exercise in patients with chronic heart failure," Chest, vol. 127, no. 3, pp. 794-802, 2005.

[47] C. B. Johnson, R. S. Beanlands, K. Yoshinaga et al., "Acute and chronic effects of continuous positive airway pressure therapy on left ventricular systolic and diastolic function in patients with obstructive sleep apnea and congestive heart failure," The Canadian Journal of Cardiology, vol. 24, no. 9, pp. 697-704, 2008.

[48] D. R. Mansfield, N. C. Gollogly, D. M. Kaye, M. Richardson, P. Bergin, and M. T. Naughton, "Controlled trial of continuous positive airway pressure in obstructive sleep apnea and heart failure," American Journal of Respiratory and Critical Care Medicine, vol. 169, no. 3, pp. 361-366, 2004.

[49] T. Kasai, S. Kasagi, K.-I. Maeno et al., "Adaptive servoventilation in cardiac function and neurohormonal status in patients with heart failure and central sleep apnea nonresponsive to continuous positive airway pressure," JACC: Heart Failure, vol. 1, no. 1, pp. 58-63, 2013.

[50] T. Kendzerska, A. S. Gershon, G. Hawker, R. S. Leung, and G. Tomlinson, "Obstructive sleep apnea and risk of cardiovascular events and all-cause mortality: a decade-long historical cohort study," PLoS Medicine, vol. 11, no. 2, Article ID e1001599, 2014.

[51] M. R. Cowie, H. Woehrle, K. Wegscheider et al., "Adaptive servo-ventilation for central sleep apnea in systolic heart failure," The New England Journal of Medicine, vol. 373, no. 12, pp. 1095-1105, 2015.

[52] R. Valle, N. Aspromonte, E. Carbonieri et al., "Fall in readmission rate for heart failure after implementation of B-type natriuretic peptide testing for discharge decision: a retrospective study," International Journal of Cardiology, vol. 126, no. 3, pp. 400-406, 2008.

[53] H. Teschler, J. Döhring, Y.-M. Wang, and M. Berthon-Jones, "Adaptive pressure support servo-ventilation: a novel treatment for Cheyne-Stokes respiration in heart failure," American Journal of Respiratory and Critical Care Medicine, vol. 164, no. 4, pp. 614-619, 2001. 
[54] A. Finckh and M. R. Tramèr, "Osteoarthritis: small studies overestimate the benefit of therapies for OA," Nature Reviews Rheumatology, vol. 6, no. 11, pp. 617-618, 2010.

[55] Z. Zhang, X. Xu, and H. Ni, "Small studies may overestimate the effect sizes in critical care meta-analyses: a metaepidemiological study," Critical Care, vol. 17, article R2, 2013. 


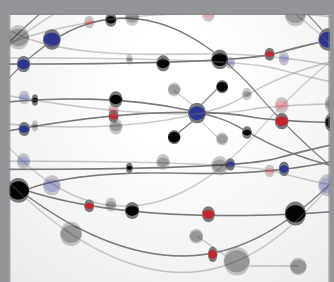

The Scientific World Journal
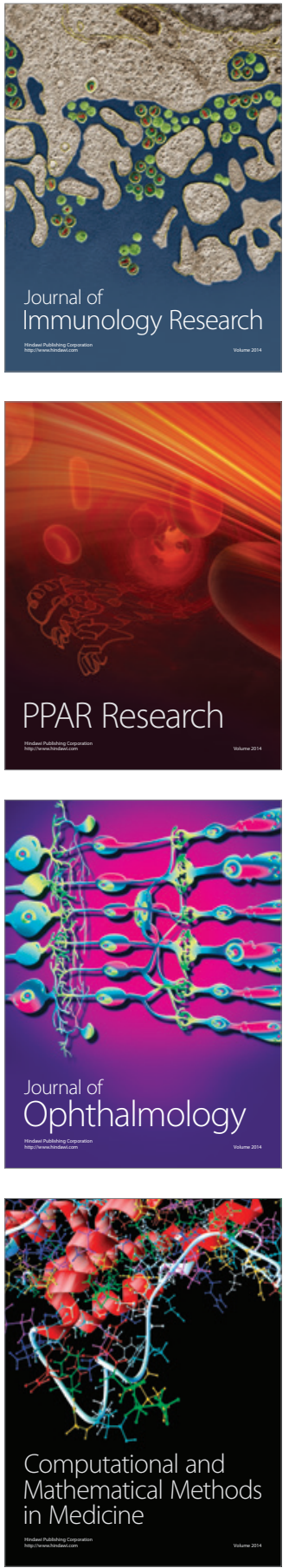

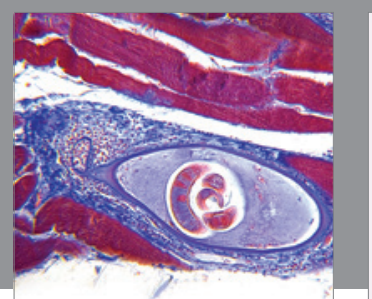

Gastroenterology Research and Practice

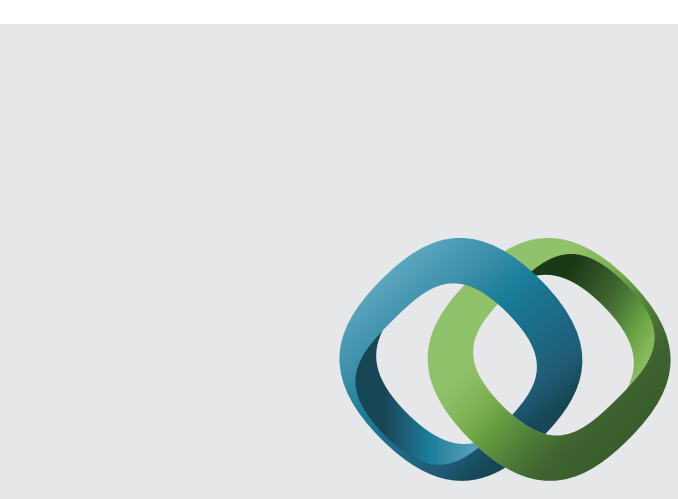

\section{Hindawi}

Submit your manuscripts at

http://www.hindawi.com
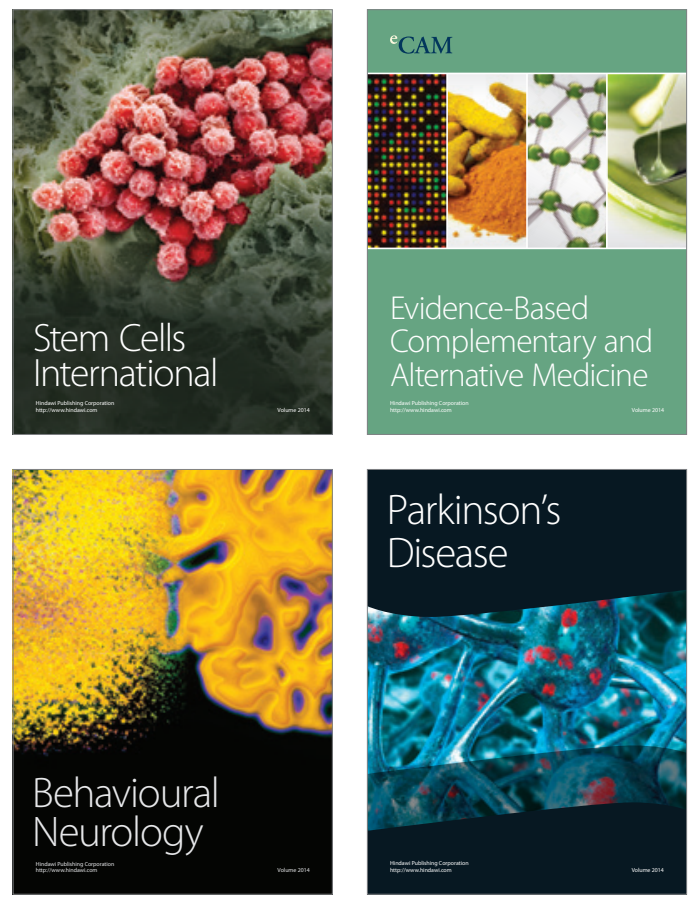
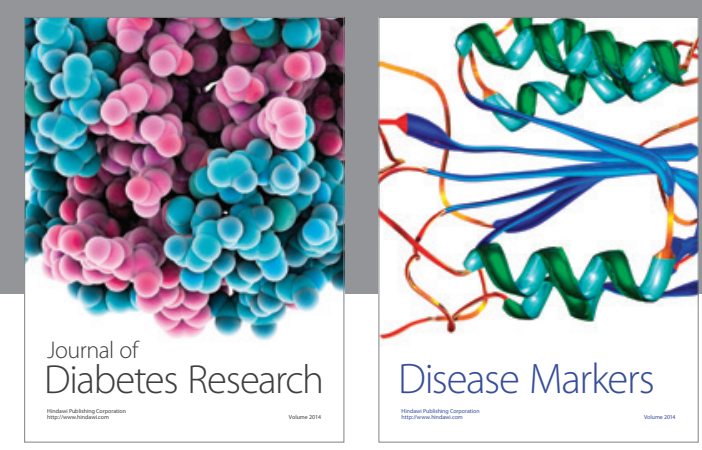

Disease Markers
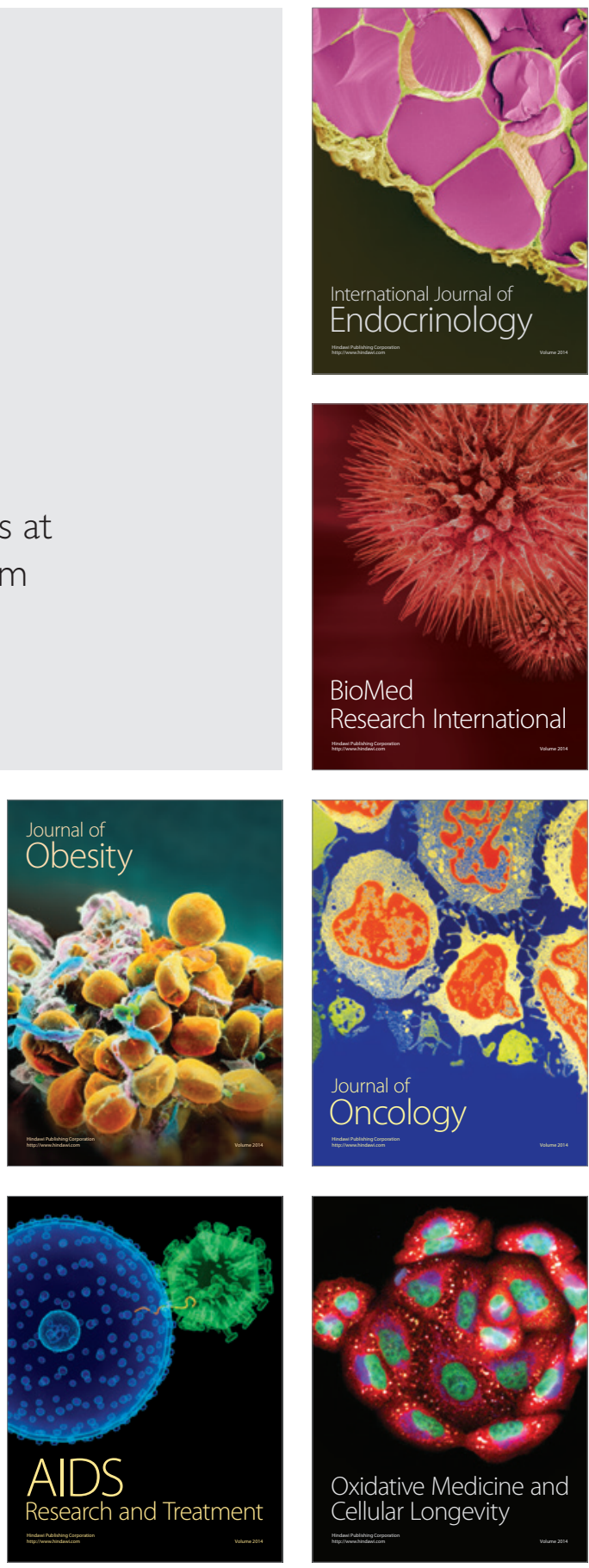